\title{
Entropy of polydisperse chains: solution on the Bethe lattice
}

\author{
Minos A. Netd* and Jürgen F. Stilck $\dagger^{\dagger}$ \\ Instituto de Física \\ Universidade Federal Fluminense \\ Av. Litorânea $s / n$ \\ 24210-346 - Niterói, RJ \\ Brazil
}

(Dated: November 21, 2018)

\begin{abstract}
We consider the entropy of polydisperse chains placed on a lattice. In particular, we study a model for equilibrium polymerization, where the polydispersivity is determined by two activities, for internal and endpoint monomers of a chain. We solve the problem exactly on a Bethe lattice with arbitrary coordination number, obtaining an expression for the entropy as a function of the density of monomers and mean molecular weight of the chains. We compare this entropy with the one for the monodisperse case, and find that the excess of entropy due to polydispersivity is identical to the one obtained for the one-dimensional case. Finally, we obtain an exponential distribution of molecular weights.
\end{abstract}

PACS numbers: $65.50 .+\mathrm{m}, 05.20 .-\mathrm{y}$

\section{INTRODUCTION}

The problem of the thermodynamic behavior of diatomic molecules which are adsorbed on two-dimensional surfaces has attracted the interest of researchers already in the thirties [1]. In the simplest model of this kind, only excluded volume interactions are taken into account, and the relevant quantity is the entropy of placing dimers (which occupy two first neighboring sites) on the lattice, which is a fundamental equation of the system. For the particular case of full occupancy and two-dimensional lattices, this problem was solved exactly by Kasteleyn and Temperley and Fisher [2]. The model may be generalized in many ways. For example, an energy may be associated to the configurations of dimers on the lattice, and such models may display rather peculiar phase transitions [3]. Also, we may generalize the athermal model allowing for chains of more than two monomers ( $M$-mers) and also considering only a fraction $\rho$ of the sites of the lattice occupied by monomers.

The entropy of $M$-mers (chains of molecular weight $M)$, as a function of the fraction of lattice sites occupied by monomers $\rho$, is a fundamental equation of this system, and thus contains all thermodynamic information. The entropy may be defined as

$$
s_{M}(\rho)=\frac{1}{V} \lim _{V \rightarrow \infty} \ln \Gamma\left(N_{p}, M ; V\right)
$$

where $\Gamma\left(N_{p}, M ; V\right)$ is the number of ways to place $N_{p}$ chains with $m$ monomers in each on the lattice with $V$ sites, and the thermodynamic limit is taken with fixed density of occupied sites $\rho=N_{p} M / V$. Besides the exact result mentioned above for $s_{2}(1)$ in two dimensions,

\footnotetext{
*Electronic address: minos@if.uff.br
}

${ }^{\dagger}$ Electronic address: jstilck@if.uff.br in the literature we may find series expansions estimates [4], closed form approximations (Bethe and Husimi lattices) [5] and transfer matrix calculations with finite size extrapolations [6] on the square lattice.

It is frequent in polymeric systems that the chains do not all have the same number of monomers, that is, a polydisperse set of chains is found. One particular model for polydisperse chains is the equilibrium polymerization model proposed by Wheeler, Kennedy and Pfeuty 7, 8] and applied to the study of equilibrium polymerization of sulfur. The system is is defined on a lattice (celular model), so that each of the $V$ cells is occupied by a monomer (a $\mathrm{S}_{8}$ ring in the case of sulfur). Each monomer may be active or inactive (open and closed rings for sulfur), and active monomers in adjacent sites may connect, forming linear polymers which are self-and mutually avoiding walks. Active monomers which are not connected to any other ones are considered one-site polymers. The statistical weight of chain composed by $m$ monomers (and therefore by $m-1$ bonds) is given by

$$
\begin{aligned}
K_{1} & \text { if } M=1, \\
2 K_{1}\left(K_{p}^{\prime}\right)^{M-1} & \text { if } M>1 .
\end{aligned}
$$

The additional factor 2 in the weight of multi-site chains is necessary for the exact correspondence of the equilibrium polymerization model to the $n$-vector of magnetism in the formal limit $n \rightarrow 0$, but it may also be justified by a combinatorial argument 8]. This model, as well as related ones, was studied in some detail in the literature [9], and experimental realizations of this situation are found in equilibrium polymerization of sulfur and in the so called living polymers [10, 11]. It is expected that for the polydisperse case the entropy is higher as compared to the monodisperse case, due to the additional degrees of freedom. Another relevant point is the distribution of molecular weights $M$. Recently the entropy of polydisperse chains and the distribution of molecular weights of the chains were calculated in the one-dimensional case 
[12]. In this paper we address these questions for the solution of the model on the Bethe lattice with coordination number $q$. As in the one-dimensional case, on the Bethe lattice it is also possible to reach closed form expressions for the entropy and for the distribution of molecular weights.

I the section II the problem is defined in more detail and the relation of the equilibrium polymerization model to a simpler version, without the presence of one-site polymers, is shown. The entropy and the distribution of molecular weights on the Bethe lattice is obtained. Final discussions and comments may be found in section III

\section{DEFINITION OF THE MODEL AND SOLUTION ON THE BETHE LATTICE}

The partition function for model for equilibrium polymerization described above may be written as:

$$
Y\left(K_{1}, K_{p}, x_{1} ; V\right)=\sum\left(2 K_{1}\right)^{N_{p}}\left(K_{p}^{\prime}\right)^{N_{b}} x_{1}^{N_{1}}
$$

where the sum is over all possible configurations of the polymers on the lattice, $N_{p}$ is the number of polymers (including one-site chains) of the configuration, $N_{b}$ corresponds to the number of bonds and $x_{1}=1 / 2$ is a factor which assures the proper counting of configurations, as discussed above. We may now perform a partial sum over configurations in this partition function. Let us consider a particular configuration of the polymers which occupy more than one site $(M>1)$. The number of monomers in internal site of the chains will be called $N_{i}$ and the number of the monomers on endpoints of the chains is $N_{e}$. The number of bonds in this configuration is $N_{b}=N_{i}+N_{e} / 2$ the total number of chains is $N_{p}=N_{1}+N_{e} / 2$ and the number of chains with more than one monomer is equal to $N_{e} / 2$. We may now write the partition function as:

$$
\begin{array}{r}
Y\left(K_{1}, K_{p}, x_{1} ; V\right)= \\
\sum^{\prime}\left(2 K_{1}\right)^{N_{e} / 2}\left(K_{p}^{\prime}\right)^{N_{e} / 2+N_{i}} \sum^{\prime \prime}\left(2 K_{1} x_{1}\right)^{N_{1}}
\end{array}
$$

where the first sum is restricted to configurations of multi-site chains and the second sum is over the configurations of the one-site polymers for a fixed configuration of the other chains. It is now trivial to perform the second sum, which is equal to $\left(1+2 K_{1} x_{1}\right)^{V-N_{i}-N_{e}}$, where we notice that $V-N_{i}-N_{e}$ is the number of sites not occupied by monomers in multi-site chains and each of these sites may be either empty (weight 1) or occupied by a one-site polymer (weight $2 K_{1} x_{1}$ ). Thus, the partition function of the equilibrium polymerization model may be written as:

$$
\begin{aligned}
Y\left(K_{1}, K_{p}, x_{1} ; V\right) & =\left(1+2 K_{1} x_{1}\right)^{V} \sum^{\prime} z_{e}^{N_{e}} z_{i}^{N_{i}} \\
& =\left(1+2 K_{1} x_{1}\right)^{V} \Xi\left(z_{e}, z_{i} ; V\right),
\end{aligned}
$$

where the sum over configurations is now restricted to chains with more than one monomers, the variable $x_{1}$ is equal to $1 / 2$ and the activities of endpoint and internal monomers are

$$
z_{e}=\frac{\sqrt{2 K_{1} K_{p}^{\prime}}}{1+2 K_{1} x_{1}}
$$

and

$$
z_{i}=\frac{K_{p}^{\prime}}{1+2 K_{1} x_{1}}
$$

respectively. We may now restrict our discussion to the model without one-site monomers, described by the grand-canonical partition function $\Xi\left(z_{e}, z_{i} ; V\right)$. In Fig. 1 a possible configuration of the chains is shown. If the lat-

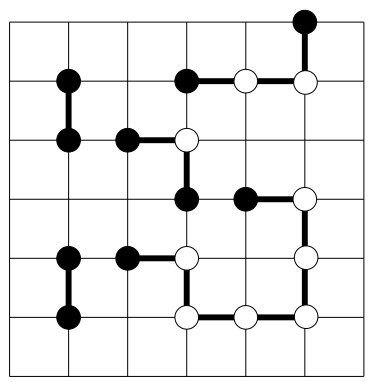

FIG. 1: A configuration of chains placed on the lattice. Internal monomers are represented as white circles and endpoint monomers are black circles. The statistical weight of this configuration with 5 chains is $z_{i}^{9} z_{e}^{10}$.

tice has $V$ sites, the density of endpoint monomers is $\rho_{e}=$ $N_{e} / V$, the density of internal monomers is $\rho_{i}=N_{i} / V$, and the total density of monomers is $\rho=\rho_{e}+\rho_{i}$. The densities may be obtained from the partition function:

$$
\rho_{i}=\frac{z_{i}}{\Xi} \frac{\partial \Xi}{\partial z_{i}}
$$

and

$$
\rho_{e}=\frac{z_{e}}{\Xi} \frac{\partial \Xi}{\partial z_{e}} .
$$

Let us now consider the model defined on a Cayley tree with coordination number $q$. Due to the hierarchical structure of this lattice, we may define partial partition functions on rooted subtrees, and it is not difficult to write down recursion relations for subtrees with an additional generation [13]. For the present model, we may define two partial partition functions $g_{0}$ and $g_{1}$, without and with a polymer bond on the root bond of the subtree, respectively. Considering the operation of attaching $\sigma=q-1 n$-generations subtrees to a new site and bond, we obtain recursion relations for the partial partition functions of a subtree with $n+1$ generations. The contributions to this recursion relations are shown 
in Fig. 2, and the resulting expressions are

$$
\begin{aligned}
& g_{0}^{\prime}=g_{0}^{\sigma}+\sigma z_{e} g_{0}^{\sigma-1} g_{1}+\frac{\sigma(\sigma-1)}{2} z_{i} g_{0}^{\sigma-2} g_{1}^{2} . \\
& g_{1}^{\prime}=z_{e} g_{0}^{\sigma}+\sigma z_{i} g_{0}^{\sigma-1} g_{1} .
\end{aligned}
$$

If we now define the ratio of partial partition functions

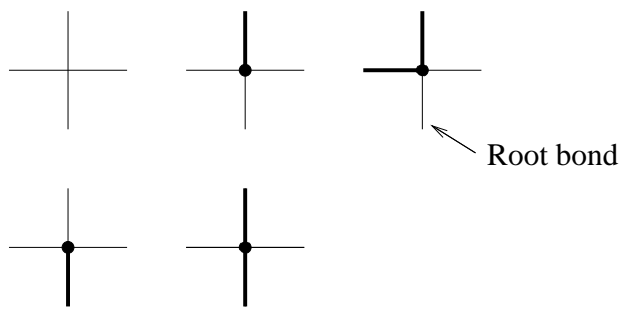

FIG. 2: Contributions to the recursion relations for the partial partition functions. The first line are the contributions to $g_{0}^{\prime}$ and the second line shows the contributions to $g_{1}^{\prime}$.

$R=g_{1} / g_{0}$, we will find the following recursion relation:

$$
R^{\prime}=\frac{z_{e}+\sigma z_{i} R}{1+\sigma z_{e} R+\frac{\sigma(\sigma-1)}{2} z_{i} R^{2}} .
$$

In the thermodynamic limit, the fixed point of this recursion relation is reached.

The partition function of the model on the Cayley tree may be obtained if we consider the operation of attaching $q$ subtrees to the central site of the tree. The result is:

$$
\Xi=g_{0}^{q}+q z_{e} g_{0}^{q-1} g_{1}+\frac{q(q-1)}{2} z_{i} g_{0}^{q-2} g_{1}^{2} .
$$

The Bethe lattice corresponds to the central region of the Cayley tree, and therefore we concentrate our attention on the densities of endpoint monomers and of internal monomers on this site. The results are:

$$
\rho_{e}=\frac{q z_{e} R}{1+q z_{e} R+\frac{q(q-1)}{2} z_{i} R^{2}},
$$

and

$$
\rho_{i}=\frac{\frac{q(q-1)}{2} z_{i} R^{2}}{1+q z_{e} R+\frac{q(q-1)}{2} z_{i} R^{2}} .
$$

For given values of the activities $z_{e}$ and $z_{i}$, the fixed point value of the ratio $R$ may be found using Eq. (13) and thus the corresponding densities of endpoint and internal monomers at the central site of the tree are defined by Eqs. (15) and (16), respectively. For $z_{e}>0$, the densities are smooth functions of the activities, but in the polymer limit (infinite chain) $z_{e} \rightarrow 0$ a continuous phase transition is found between a non-polymerized phase $\rho_{i}=0$ and a polymerized phase $\rho_{i}>0$, at $z_{i}=1 /(q-1)$ [14, 15].

If we call $s\left(\rho_{e}, \rho_{i}\right)$ the entropy per site of the model, we notice that the ratios of the chemical potentials and $k_{B} T$ are given by

$$
\frac{\mu_{e}}{k_{B} T}=\ln z_{e}=-\left(\frac{\partial s}{\partial \rho_{e}}\right)_{\rho_{i}},
$$

and

$$
\frac{\mu_{i}}{k_{B} T}=\ln z_{i}=-\left(\frac{\partial s}{\partial \rho_{i}}\right)_{\rho_{e}} .
$$

We then may obtain the entropy integrating the chemical potentials. To do this, we start inverting equations for the densities (15) and (16), which leads to:

$$
z_{e}=\frac{\rho_{e}}{q\left(1-\rho_{i}-\rho_{e}\right) R},
$$

and

$$
z_{i}=\frac{2 \rho_{i}}{q(q-1)\left(1-\rho_{i}-\rho_{e}\right) R^{2}} .
$$

If these activities are now substituted into the fixed point equation $R^{\prime}=R$, we obtain, from Eq. (13) the fixed point value of the ratio:

$$
R^{2}=\frac{2 \rho_{i}+\rho_{e}}{q-2 \rho_{i}-\rho_{e}} .
$$

Substituting this result in Eqs. (19) and (20), we obtain the activities as functions of the densities in the thermodynamic limit:

$$
z_{e}=\frac{\rho_{e} \sqrt{q-2 \rho_{i}-\rho_{e}}}{q\left(1-\rho_{i}-\rho_{e}\right) \sqrt{2 \rho_{i}+\rho_{e}}}
$$

and

$$
z_{i}=\frac{2 \rho_{i}\left(q-2 \rho_{i}-\rho_{e}\right)}{q(q-1)\left(1-\rho_{i}-\rho_{e}\right)\left(2 \rho_{i}+\rho_{e}\right)} .
$$

The entropy may now be calculated integrating the Eqs. (17) and (18) in the $\left(\rho_{e}, \rho_{i}\right)$ plane. We choose the trajectory $(0,0) \rightarrow\left(0, \rho_{i}\right) \rightarrow\left(\rho_{e}, \rho_{i}\right)$, so that:

$$
\begin{aligned}
s\left(\rho_{e}, \rho_{i}\right)= & -\int_{0}^{\rho_{i}} \ln \left[\frac{q-2 \rho}{q(q-1)(1-\rho)}\right] d \rho- \\
& \int_{0}^{\rho_{e}} \ln \left[\frac{\rho \sqrt{q-2 \rho_{i}-\rho}}{q\left(1-\rho_{i}-\rho\right) \sqrt{2 \rho_{i}+\rho}}\right] d \rho .
\end{aligned}
$$

The result of this integration is:

$$
\begin{aligned}
s\left(\rho_{e}, \rho_{i}\right)= & -\frac{1}{2}\left(q-2 \rho_{i}\right) \ln q+\rho_{i} \ln (q-1)-\rho_{e} \ln \frac{\rho_{e}}{q}+ \\
& \frac{1}{2}\left(q-2 \rho_{i}-\rho_{e}\right) \ln \left(q-2 \rho_{i}-\rho_{e}\right)- \\
& \left(1-\rho_{i}-\rho_{e}\right) \ln \left(1-\rho_{i}-\rho_{e}\right)+ \\
& \frac{1}{2}\left(2 \rho_{i}+\rho_{e}\right) \ln \left(2 \rho_{i}+\rho_{e}\right)-\rho_{i} \ln \left(2 \rho_{i}\right) .
\end{aligned}
$$

The entropy may be compared to results in the literature in some particular cases. For $\rho_{e}=0$ the case of infinite polymers is obtained, and the expression reduces to Eq. (26) in reference [5]. The entropy of dimers on the Bethe lattice (Eq. (27) in reference [5], where in the correct result $\rho z$ should be replaced by $\rho / z)$ is obtained 
for $\rho_{i}=0$. Finally, the one-dimensional result (Eq. (9) in reference [12]) is recovered for $q=2$. To compare the result with the monodisperse case, it is convenient to rewrite the entropy Eq. (25) as a function of the mean molecular weight $\bar{M}=2\left(1+\rho_{i} / \rho_{e}\right)$ and the total density of monomers $\rho=\rho_{i}+\rho_{e}$. The resulting expression is:

$$
\begin{aligned}
s_{\bar{M}}(\rho)= & \frac{\rho}{\bar{M}} \ln q+\rho \frac{\bar{M}-2}{\bar{M}} \ln (q-1)-\frac{2 \rho}{\bar{M}} \ln \frac{2 \rho}{\bar{M}}+ \\
& \left(\frac{q}{2}-\rho \frac{\bar{M}-1}{\bar{M}}\right) \ln \left(1-2 \rho \frac{\bar{M}-1}{q \bar{M}}\right)- \\
& (1-\rho) \ln (1-\rho)+\rho \frac{\bar{M}-1}{\bar{M}} \ln \left(2 \rho \frac{\bar{M}-1}{\bar{M}}\right)- \\
& \rho \frac{\bar{M}-2}{\bar{M}} \ln \left(\rho \frac{\bar{M}-2}{\bar{M}}\right) .
\end{aligned}
$$

We may now find the contribution to the entropy of polydispersivity, calculating $\Delta s_{M}(\rho)=s_{\bar{M}}(\rho)-s_{M}(\rho)$ for $\bar{M}=M$, where $s_{M}(\rho)$ is the entropy of the monodisperse case (Eq. (22) of reference [5]). We find a result which is independent of $q$ :

$$
\Delta s_{M}(\rho)=\frac{\rho}{M}[(M-1) \ln (M-1)-(M-2) \ln (M-2)],
$$

and therefore this result is identical to the one obtained in the one-dimensional case studied in reference [12].

Finally, we may find the distribution of the molecular weights in the polydisperse case. To obtain this result, we must keep track of the number of monomers incorporated into each chain, and we may define multiple subtree partial partition functions $g_{M}, M=0,1, \ldots$, in such a way that $g_{0}$ corresponds to a subtree with no polymer bond at the root, as before, and $g_{M}$ stands for a subtree with a polymer bond on the root connected to $M$ monomers above. The recursion relations for these partial partition functions will be:

$$
\begin{aligned}
g_{0}^{\prime}= & g_{0}^{\sigma}+\sigma z_{e} g_{0}^{\sigma-1} \sum_{M=1}^{\infty} g_{M}+ \\
& \frac{\sigma(\sigma-1)}{2} z_{i} g_{0}^{\sigma-2} \sum_{M, N=1}^{\infty} g_{M} g_{N}, \\
g_{1}^{\prime}= & z_{e} g_{0}^{\sigma}, \\
g_{M}^{\prime}= & \sigma z_{i} g_{0}^{\sigma-1} g_{M-1}, M=2,3, \ldots
\end{aligned}
$$

We proceed defining the ratios of partial partition functions $R_{M}=g_{M} / g_{0}$, for $M=1,2, \ldots$. The recursion relations for the ratios will be:

$$
\begin{aligned}
R_{1}^{\prime} & =\frac{z_{e}}{D}, \\
R_{M}^{\prime} & =\frac{\sigma z_{i} R_{M-1}}{D}, M=2,3, \ldots,
\end{aligned}
$$

where

$$
D=1+\sigma z_{e} \sum_{M=1}^{\infty} R_{M}+\frac{\sigma(\sigma-1)}{2} z_{i} \sum_{M, N=1}^{\infty} R_{M} R_{N}
$$

Inspection of these set of recursion relations leads to the following Ansatz for the fixed point values of the ratios $R_{M}=\alpha^{M-1} R_{1}$, where $\alpha=R_{1} \sigma z_{i} / z_{e}$. The ratios $R_{M}$ are related to the ratio $R$ defined above through $R=$ $\sum_{M=1}^{\infty} R_{M}=R_{1} \sum_{M=1}^{\infty} \alpha^{M-1}=R_{1} /(1-\alpha)$. Using the fixed point value for $R$ found in Eq. (21), we may then find the values of $R_{1}$ and $\alpha$ in the thermodynamic limit. The results are:

$$
R_{1}=\frac{\rho_{e}}{\sqrt{\left(\rho_{e}+2 \rho_{i}\right)\left(q-\rho_{e}-2 \rho_{i}\right)}},
$$

and

$$
\alpha=\frac{2 \rho_{i}}{\rho_{e}+2 \rho_{i}} .
$$

Now we consider the operation of attaching $q$ subtrees to the central site of the tree. The probability that an endpoint of a chain with $M$ monomers is located on the central site will be equal to $q z_{e} R_{M-1}$, and the probability to have an endpoint of a chain located on the central site is equal to $q z_{e} R$. Therefore, the probability to find a chain with exactly $M$ monomers among all chains on the lattice with mean molecular weight $\bar{M}$ will be given by:

$$
\begin{aligned}
r_{M}= & \frac{R_{M-1}}{R}=(1-\alpha) \alpha^{M-2}= \\
& \frac{1}{\bar{M}-1}\left(\frac{\bar{M}-2}{\bar{M}-1}\right)^{M-2} .
\end{aligned}
$$

This exponential distribution of molecular weights is again independent of the coordination number $q$ and therefore is identical to the one obtained in [12] for the one-dimensional case $q=2$.

\section{FINAL DISCUSSIONS AND COMMENTS}

Although the equilibrium polymerization model was extensively studied in the literature, the main focus of these studies was its critical behavior, which occurs in the limit $K_{1} \rightarrow 0\left(z_{e} \rightarrow 0\right)$, where the mean molecular weight of the chains diverges. Here, similarly to what was done by Dudowicz et al [9] for a similar model, which essential differs from the present one only by the fact that it is canonical with respect to the iniciator molecules, we turn our attention to the region of parameters where the model is non critical. The calculations performed by Dudowicz et al were in the Flory-Huggins approximation, which for simple polymer models is equivalent to the Bethe lattice solution. It is therefore not surprising that boths calculations lead to an exponential distribution of molecular weights of the chains. The model studied by Dudowicz et al is canononical with respect to the initiator molecules, while the present model is grand-canonical with respect to the endpoint monomers. In the polymer limit, the distribution of molecular weights should in general be described by the scaling behavior [16]

$$
r_{M} \approx M^{\gamma-1} \exp (-M)
$$


in the limit of small overlap, reducing to an exponential decay at large overlaps. The critical exponent $\gamma$ is in general larger than 1, but its classical value is 1 . Since the Flory-Huggins approximation and Bethe lattice calculations should lead to classical exponents, the exponential decay of the distribution of molecular weights is expected in the polymer limit $\bar{M} \rightarrow \infty$, but nonexponential decay would be allowed for finite $\bar{M}$. We are presently innvestigating if this actually occurs when the model is studied using better (mean field) approximations.

Although the details of the calculations above were presented for the restricted equilibrium polymerization model, without the presence of one-site chains, it is easy to obtain the correpondin results for the original model, using Eqs. (6), (7) and (8). For example, the density of sites occupied by the one-site polymers is

$$
\rho_{1}=\frac{x_{1}}{Y} \frac{\partial Y}{\partial x_{1}}
$$

making $x_{1}=1 / 2$. The result is

$$
\rho_{1}=\frac{K_{1}}{1+K_{1}}\left(1-\rho_{e}-\rho_{i}\right) .
$$

[1] R. H. Fowler e G. S. Rushbrooke, Trans. Faraday Soc. 33, 1272 (1937).

[2] P. W. Kasteleyn, Physica 27, 1209 (1961); H. N. V. Temperley e M. E. Fisher, Philos. Mag. 6, 1061 (1961); M. E. Fisher, Phys. Rev. 124, 1664 (1961).

[3] J. F. Nagle, C. S. O. Yokoi, and S. M. Battacharjee, in Phase Transitions and Critical Phenomena, edited by C. Domb and J.L. Lebowitz (Academic, London, 1989), Vol. 13.

[4] A. M. Nemirovsky and M. D. Coutinho-Filho, Phys. Rev. A 39, 3120 (1989).

[5] J. F. Stilck and M. J. de Oliveira, Phys. Rev. A 42, 5955 (1990).

[6] W. G. Dantas and J. F. Stilck, Phys. Rev. E 67, 031803 (2003); W. G. Dantas, M. J. de Oliveira and J. F. Stilck, Phys. Rev. E 76, 031133 (2007).

[7] J. C. Wheeler, S. J. Kennedy and P. Pfeuty, Phys. Rev.
A similar calculation may be done for the density of polymers (including the one-site chains) $\rho_{p}=N_{p} / N$, leading to:

$$
\rho_{p}=\frac{K_{1}}{1+K_{1}}\left(1+\frac{1-K_{1}}{2 K_{1}} \rho_{e}-\rho_{i},\right)
$$

while the density of bonds is given by:

$$
\rho_{b}=\frac{\rho_{e}}{2}+\rho_{i}
$$

\section{Acknowledgements}

MAN acknowledges a doctoral grant from the brazilian agency $\mathrm{CNPq}$ and JFS thanks the same agency for partial financial assistance.

Lett. 45, 1748 (1980);

[8] J. C. Wheeler and P. Pfeuty, Phys. Rev. A 24, 1050 (1981).

[9] J. Dudowicz, K. F. Freed and J. F. Douglas, J. Chem. Phys. 111, 7116 (1999).

[10] S. C. Greer, J. Phys. Chem B 102, 5413 (1998).

[11] P. M. Pfeuty and J. C. Wheeler, Phys. Rev. A 27, 2178 (1983).

[12] J. F. Stilck, M. A. Neto, and W. G. Dantas, Physica A 368, 442 (2006).

[13] R. Baxter, Exactly solved models in statistical mechanics, (1982).

[14] P. Gujrati, Phys. Rev. Lett. 53, 2453 (1984).

[15] J. F. Stilck and J. C. Wheeler, J. Stat. Phys. 46, 1 (1987).

[16] L. Shäfer, Phys. Rev. B 46, 6061 (1992). 